\title{
ARTICLE
}

Received 2 Oct 2013 | Accepted 3 Dec 2013 | Published 9 Jan $2014 \quad$ DOI: 10.1038/ncomms4048

\section{Evidence for spin selectivity of triplet pairs in superconducting spin valves}

\author{
N. Banerjee ${ }^{1}$, C.B. Smiet ${ }^{2}$, R.G.J. Smits ${ }^{3}$, A. Ozaeta ${ }^{4}$, F.S. Bergeret ${ }^{4}$, M.G. Blamire ${ }^{1} \&$ J.W.A. Robinson ${ }^{1}$
}

Spin selectivity in a ferromagnet results from a difference in the density of up- and down-spin electrons at the Fermi energy as a consequence of which the scattering rates depend on the spin orientation of the electrons. This property is utilized in spintronics to control the flow of electrons by ferromagnets in a ferromagnet (F1)/normal metal (N)/ferromagnet (F2) spin valve, where $\mathrm{F} 1$ acts as the polarizer and $\mathrm{F} 2$ the analyser. The feasibility of superconducting spintronics depends on the spin sensitivity of ferromagnets to the spin of the equal spintriplet Cooper pairs, which arise in superconductor (S)-ferromagnet (F) heterostructures with magnetic inhomogeneity at the S-F interface. Here we report a critical temperature dependence on magnetic configuration in current-in-plane F-S-F spin valves with a holmium spin mixer at the $\mathrm{S}-\mathrm{F}$ interface providing evidence of a spin selectivity of the ferromagnets to the spin of the triplet Cooper pairs.

\footnotetext{
${ }^{1}$ Department of Materials Science and Metallurgy, University of Cambridge, 27 Charles Babbage Road, Cambridge CB3 OFS, UK. ${ }^{2}$ Huygens Laboratory, Leiden University, PO Box 9504, 2300 RA Leiden, The Netherlands. ${ }^{3}$ MESA + Institute for Nanotechnology, University of Twente, 7500 AE Enschede, The Netherlands. ${ }^{4}$ Centro de Física de Materiales (CFM-MPC), Centro Mixto CSIC-UPV/EHU, Manuel de Lardizabal 4, E-20018 San Sebastián, Spain. Correspondence and requests for materials should be addressed to J.W.A.R. (email: jjr33@cam.ac.uk).
} 
$\mathrm{T}$ he dependence of the critical temperature $\left(T_{\mathrm{C}}\right)$ of ferromagnet-superconductor (F-S) bilayers has its origin in the spatially oscillating components of the wave function of the singlet Cooper pairs induced by the exchange field in the $\mathrm{F}$ layers ${ }^{1}$. The exchange field introduces a momentum mismatch between the spin-up and spin-down electrons that form a singlet pair, and this results in a weak oscillatory dependence superimposed on the decrease of $T_{\mathrm{C}}$ with increasing thickness of the $\mathrm{F}$ layer $^{2-4}$. In an $\mathrm{F}-\mathrm{S}-\mathrm{F}$ spin valve, the reversal of one $\mathrm{F}$ layer modifies the spatial properties of the decaying oscillation, resulting in a spin-switch effect in which the $T_{\mathrm{C}}$ is greater when the $\mathrm{F}$ layer moments are antiparallel (AP) than when they are parallel $(\mathrm{P})^{5-7}$. This (standard) spin-switch effect has been experimentally demonstrated ${ }^{8,9}$ and the difference in $T_{\mathrm{C}}$ between $\mathrm{P}$ and AP configurations of an $\mathrm{F}-\mathrm{S}-\mathrm{F}$ spin valve can be understood as follows: in the AP state, the pair-breaking effect of the $\mathrm{F}$ layers is reduced, as the net ferromagnetic exchange field of the structure is partially compensated, meaning $T_{C}$ is maximized ${ }^{1}$; for a $P$ state, the pair-breaking effect is maximized, thus $T_{\mathrm{C}}$ is reduced.

This behaviour should be substantially modified in S-F systems in which conversion between singlet pairs and odd-frequency spin-one triplet pairs is possible, for example, by introducing magnetic non-collinearity (that is, a spin-mixer layer) at the S-F interface $^{10,11}$. As spin-one triplet pairs are immune to pair breaking by the exchange field in $\mathrm{F}$, the proximity effect coupling between $\mathrm{S}$ and $\mathrm{F}$ layers is enhanced. As with the conventional superconductor-normal metal proximity effect, the increased 'leakage' of pairs from the $S$ layer should reduce the singlet pair amplitude within it and hence decrease the $T_{\mathrm{C}}$ of the structure ${ }^{12}$. A number of experiments have been performed to test these predictions: for example, in S-F'-F structures, both Leksin et al. ${ }^{13}$ and Zdravkov et al. ${ }^{14}$ recently demonstrated a minimum in $T_{\mathrm{C}}$ when the $\mathrm{F}$ and $\mathrm{F}^{\prime}$ layers were orthogonal-the configuration that theoretically maximizes singlet-triplet pair conversion.

More direct evidence for the generation of triplet pairing was obtained from S-F-S Josephson junctions in which, if interfacial spin-mixer layers were present, supercurrents could be measured through F-layer thicknesses much larger than the singlet coherence length ${ }^{15-23}$. As spin-one triplet pairs, unlike singlet pairs, can carry spin, these results mean that combining superconducting and spin electronics (superconducting spintronics) opens up real potential for practical lowtemperature applications ${ }^{24}$.

Conventional spintronics relies on the spin selectivity of ferromagnets, which originates from the difference between the spin-up and spin-down density of states at the Fermi level. By extension, the realization of superconducting spintronics requires a selectivity between spin up-up and spin down-down triplet pairs; however, experiments performed to date are not spin sensitive and the polarization of a triplet supercurrent is so far unknown.

In this article we report experimental results that demonstrate a $T_{\mathrm{C}}$ dependence on the magnetic configuration in current-inplane F-S-F spin valves, which incorporate a Ho spin-mixer layer at the S-F interface, providing the first evidence for a sensitivity to triplet pair spin direction in a ferromagnet. The experiments were performed on $\mathrm{Py}(8 \mathrm{~nm}) / \mathrm{Ho}\left(d_{\mathrm{Ho}}\right) / \mathrm{Nb}\left(d_{\mathrm{Nb}}\right) / \mathrm{Ho}\left(d_{\mathrm{Ho}}\right) / \mathrm{Py}$ $(5 \mathrm{~nm}) / \mathrm{FeMn}(5 \mathrm{~nm})$ heterostructures as sketched in Fig. 1a. The bottom Py $\left(\mathrm{Ni}_{80} \mathrm{Fe}_{20}\right)$ layer is pinned by exchange bias to an antiferromagnetic layer of FeMn, whereas the orientation of the free (top) Py layer can be switched by applying an in-plane magnetic field that is greater than its coercive field so that $\mathrm{P}$ or AP states can be achieved. The $\mathrm{Nb}$ is interfaced by $\mathrm{Ho}$, as this rareearth helimagnet has previously been shown by our group ${ }^{18}$ and by Sosnin et al. ${ }^{25}$ to be a spin-mixer. The stacks were capped with 4-nm-thick layer of (non-superconducting) $\mathrm{Nb}$ to prevent a
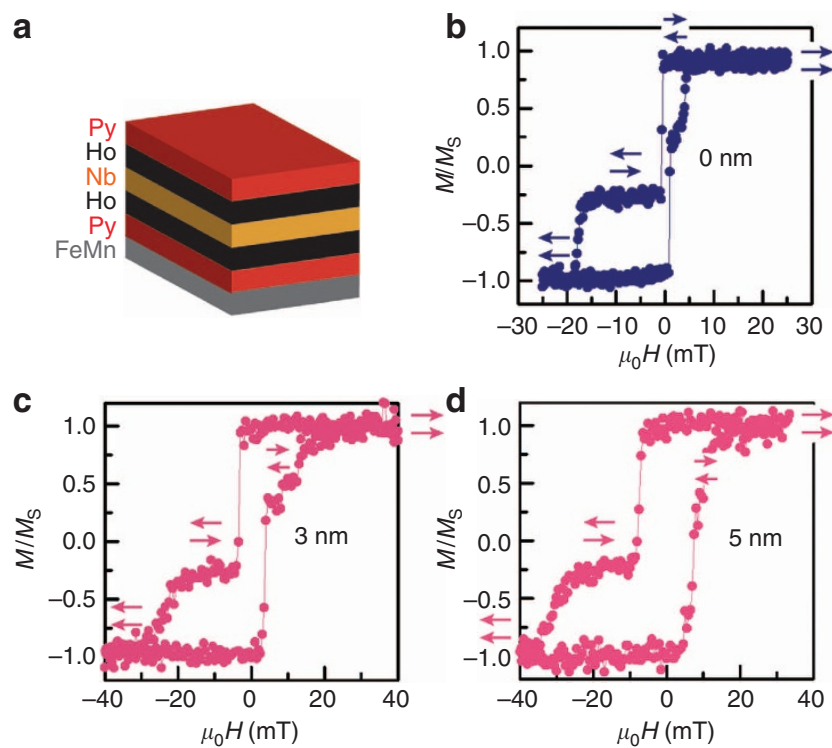

Figure 1 | Structure and magnetic characterization of the superconducting spin valves. (a) An illustration of a $\mathrm{Py}(8 \mathrm{~nm}) / \mathrm{Ho} / \mathrm{Nb} /$ $\mathrm{Ho} / \mathrm{Py}(5 \mathrm{~nm}) / \mathrm{FeMn}(5 \mathrm{~nm})$ spin valve. The spin valves were cooled in a positive field to $20 \mathrm{~K}$ to obtain the magnetization versus in plane magnetic field loops ( $M-H$ loops): (b) $M-H$ loop for a spin valve without any Ho layers; (c) $\mathrm{M}-\mathrm{H}$ loop for a spin valve with 3-nm-thick layer of Ho on either side of $\mathrm{Nb}$; and (d) $\mathrm{M}-\mathrm{H}$ loop for a spin valve with a 5 -nm-thick layer of $\mathrm{Ho}$ on either side of $\mathrm{Nb}$. Arrows in $\mathbf{b}-\mathbf{d}$ indicate the probable magnetic configuration of the Py layers in the spin valves.

oxidation of the top Py layer. The central $\mathrm{Nb}$ layer thickness $\left(d_{\mathrm{Nb}}\right)$ was varied to achieve a superconducting transition in the $2-7 \mathrm{~K}$ range. The thicknesses of the Ho layers $\left(d_{\mathrm{Ho}}\right)$ were varied in the $0-7 \mathrm{~nm}$ range.

\section{Results}

Magnetic characterization of superconducting spin valves. In Fig. $1 \mathrm{~b}-\mathrm{d}$, we have plotted the magnetization versus in-plane magnetic field $(M-H)$ for several spin valves with different Ho layer thicknesses, at temperatures close to $20 \mathrm{~K}$, measured using a cryogen-free vibrating sample magnetometer. The $M-H$ loops show that at high positive fields the spin valves are in a $\mathrm{P}$ state, and as the magnetic field direction is reversed the free Py layer switches and a stable AP state is obtained. On increasing the negative field, the exchanged-biased Py layer eventually switches and hence the spin valves return to the P state. The increase in coercivity of both Py layers and a reduction in the field range of the AP state as a function of Ho layer thickness suggest an exchange coupling at the $\mathrm{Ho} / \mathrm{Py}$ interfaces.

Transport measurements in the superconducting transition. Magnetic field- and temperature-dependent resistance measurements of unpatterned samples were performed at temperatures around the superconducting transition using a four-point current-bias technique in a pulse-tube cryocooled measurement system at a constant current of $200 \mu \mathrm{A}$. We investigated the behaviour of the spin valves with several different $\mathrm{Ho}$ and $\mathrm{Nb}$ layer thicknesses by measuring the resistance $(R)$ of the structures in both the $\mathrm{P}$ and AP states by sweeping the applied field $(H)$ from positive to negative values and back again. For comparison, we also measured $R$ as a function of temperature $T$ in the superconducting transition for both the P and AP states. However, because the effect of the magnetic state on $T_{\mathrm{C}}$ is in the $\mathrm{mK}$ 
range, $\mathrm{R}-\mathrm{H}$ at constant temperature was found to be a more reliable probe of the superconducting state and its dependence on magnetic configuration.

Figure 2 shows $R-H$ data from different samples with different $\mathrm{Nb}$ and Ho layer thicknesses. We first focus on the plots in Fig. 2a,c where each spin valve contains Ho layers. The different lines represent measurements taken at different temperatures within the resistive transition of the sample and no offset has been added in plotting the data: each curve consists of a forward and a backward field sweep. The data show a pronounced, but temperature-dependent, peak in $R$ at the fields corresponding to the AP state (indicated by arrows) meaning that, in all cases, the $T_{\mathrm{C}}$ is lower in the AP state than in the P state (that is, the inverse of the standard spin-valve effect discussed in the Introduction). The corresponding change in resistance for each curve (called 'magnetoresistance' here or 'MR') is plotted in Fig. 2b,d, where $\mathrm{MR}$ is given by $(R(\mathrm{AP})-R(\mathrm{P})) / R(\mathrm{P})$. The $\mathrm{MR}$ increases as the temperature decreases with no $M R$ above $T_{C}$. This implies that the $\mathrm{MR}$ is related to a change in $T_{\mathrm{C}}$ induced by the magnetic state of the valve and is not related to conventional MR effects due to differential scattering of spin-up and spin-down electrons in the Py layers.

We have also directly measured the $R-T$ dependence at zero field for $\mathrm{P}$ and AP orientations (an example is shown in Fig. 2b where $T_{\mathrm{C}}(\mathrm{P})$ is of the order of $10 \mathrm{mK}$ larger than $T_{\mathrm{C}}(\mathrm{AP})$. The $\mathrm{P}$ state was set by applying a positive saturation field followed by a reduction to zero field, or in the AP state by first applying a large positive field, ramping to $-15 \mathrm{mT}$ and then to zero field.
The behaviour of these structures is the inverse of the standard behaviour seen for simple F-S-F structures without spin-mixer layers ${ }^{8,9}$. To confirm this, we have also measured structures without any Ho as shown in Fig. 2e,f. To achieve a measurable $T_{C}$, the thickness of the $\mathrm{Nb}$ layer was increased to $32 \mathrm{~nm}$ to compensate the additional suppression of $T_{C}$, as the Py layers are in direct contact with $\mathrm{Nb}$. The pink curve shows $R$ as the field is swept from positive to negative and shows a clear dip from around -1 to $-18 \mathrm{mT}$, and the reverse scan (blue curve) shows a dip of similar magnitude from 1 to $4 \mathrm{mT}$; thus, a standard spinswitch effect is observed. The temperature was highly stable throughout this measurement as shown by the black and grey data in Fig. 2e, which shows $T$ versus $H$.

To summarize, the data in Fig. 2 shows two behaviours: in the absence of Ho, a standard spin-valve effect is observed but the addition of Ho results in an inverse spin-valve effect in which the $T_{\mathrm{C}}$ is enhanced when the Py layers are P rather than AP. We have also verified the inverse spin-valve effect in valves with different thicknesses of Ho $(2,3$ and $7 \mathrm{~nm})$-example data are shown in Fig. 3a,b for Ho thicknesses of 2 and $7 \mathrm{~nm}$.

Asymmetry in magnetization and resistance versus field loops. Figure $2 \mathrm{e}$ (no Ho) shows a pronounced asymmetry in the downward peak widths of the $R-H$ plots for the forward (from negative to positive) and reverse (positive to negative) field sweep directions. This asymmetry is expected and can be explained with reference to the corresponding $M-H$ loop of the spin valve shown
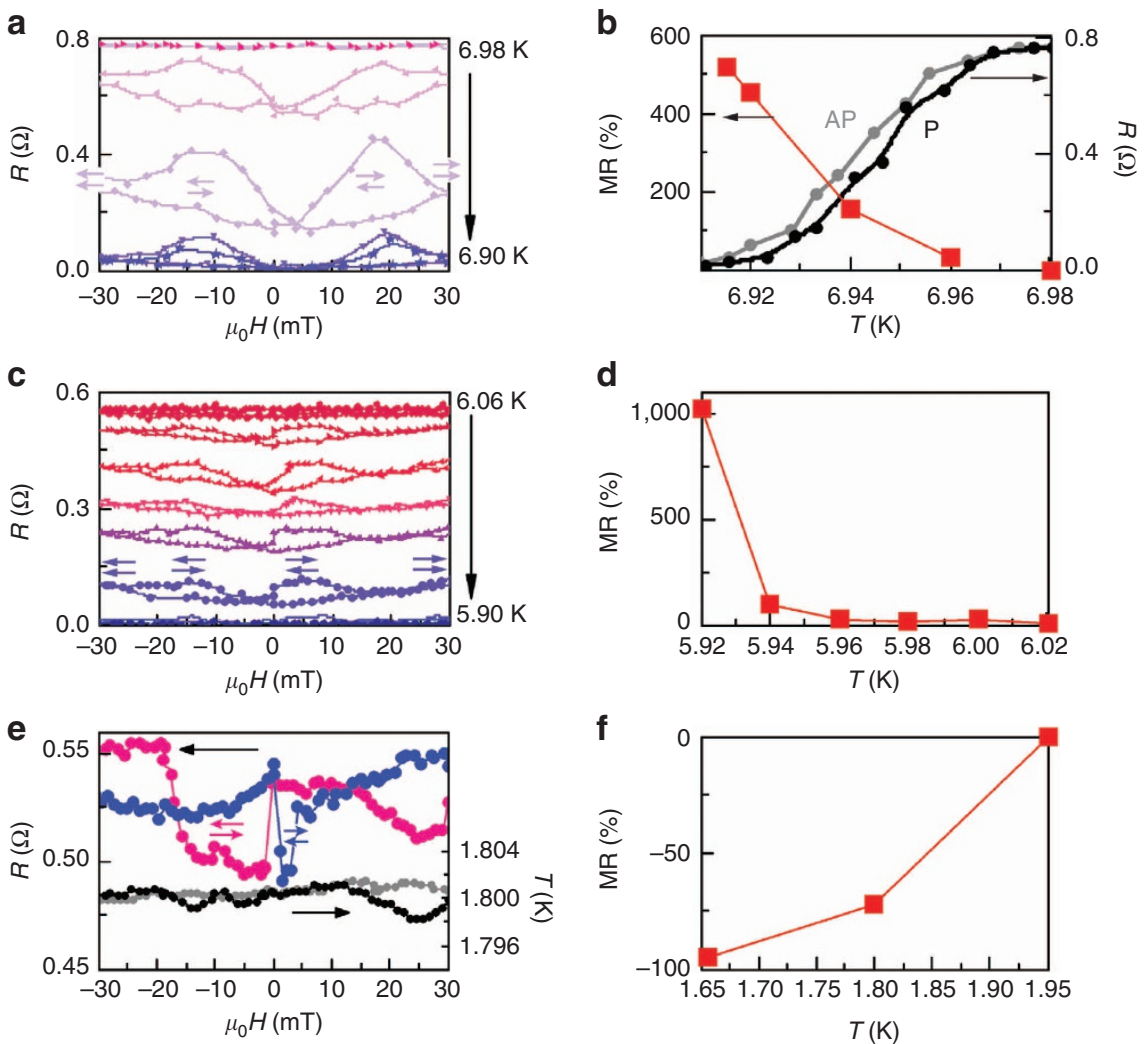

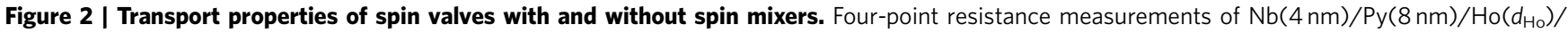
$\mathrm{Nb}\left(d_{\mathrm{Nb}}\right) / \mathrm{Ho}\left(d_{\mathrm{Ho}}\right) / \mathrm{Py}(5 \mathrm{~nm}) / \mathrm{FeMn}(5 \mathrm{~nm})$ spin valves in the superconducting transition. (a) $R-H$ for 5 -nm-thick top and bottom Ho layers and a 26 -nmthick Nb layer; (b) MR versus $T$ and also $R-T$ data for the same spin valve for parallel (black data points and black solid line) and antiparallel (grey data points and grey solid line) configurations of the Py layers. (c) $R-H$ and (d) MR versus $T$ for 5-nm-thick Ho layers and a 20-nm-thick Nb layer. (e) $\mathrm{R}-\mathrm{H}$ for a 32-nm-thick layer of $\mathrm{Nb}$ and no $\mathrm{Ho}$, and the corresponding variation of temperature $T$ on $H$ (demonstrating the stability of $T$ during the measurement of $R-H)$. (f) MR versus $T$ for the spin valve without Ho. Coloured arrows in $\mathbf{a}, \mathbf{c}, \mathbf{e}$ indicate the most probable magnetic configuration of the Py layers in the spin valves. Horizontal black arrows in $\mathbf{b}$ and $\mathbf{e}$ indicate the corresponding $y$ axis to which the plots correspond to. 


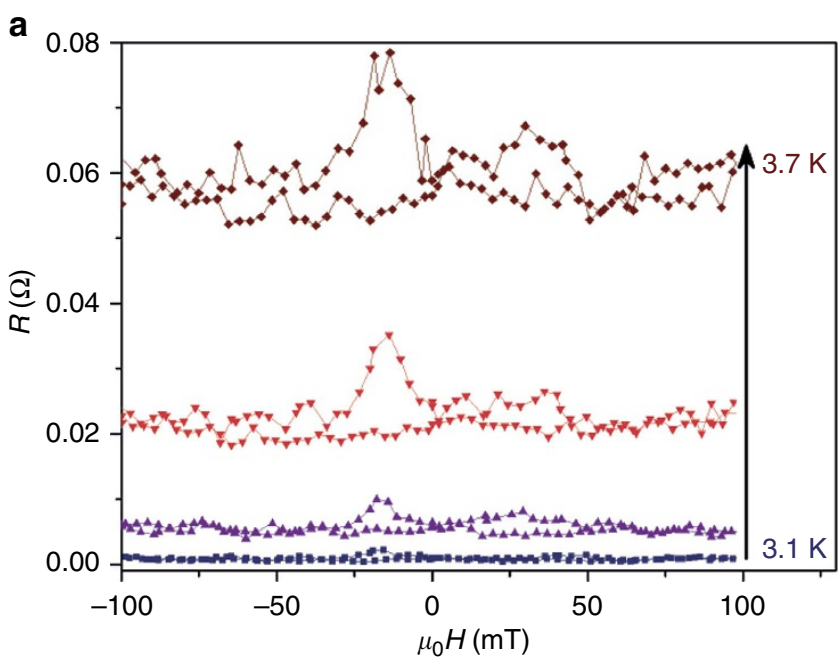

b

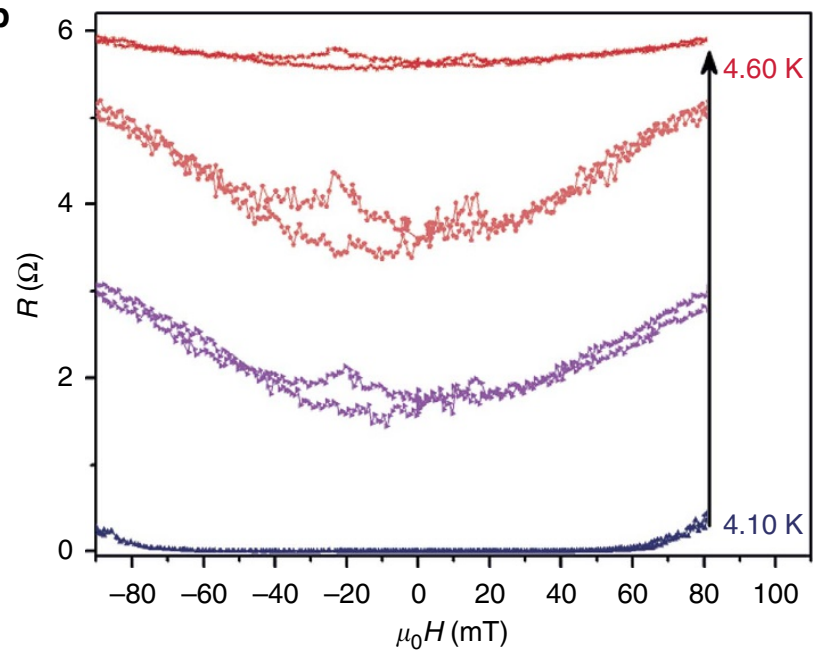

Figure 3 | Resistance-field measurements for different spin-mixer layer thicknesses. Four-point resistance measurements of $\mathrm{Nb}(4 \mathrm{~nm}) / \mathrm{Py}(8 \mathrm{~nm}) /$ $\mathrm{Ho}\left(d_{\mathrm{Ho}_{\mathrm{O}}}\right) / \mathrm{Nb}\left(d_{\mathrm{Nb}}\right) / \mathrm{Ho}\left(d_{\mathrm{Ho}_{\mathrm{O}}}\right) / \mathrm{Py}(5 \mathrm{~nm}) / \mathrm{FeMn}(5 \mathrm{~nm})$ spin valves in the superconducting transition. (a) $\mathrm{R}-\mathrm{H}$ for 2-nm-thick top and bottom $\mathrm{Ho}$ layers and 26-nm-thick Nb layer. (b) $\mathrm{R}-\mathrm{H}$ for 7 -nm-thick Ho layers and 26-nm-thick Nb layer.

in Fig. 1b, where the exchange bias of the bottom $5 \mathrm{~nm}$ Py layer by FeMn leads to an AP state that is stable over a wide magnetic field range. This asymmetry in the switching field is also manifested in the $R-H$, where in the reverse field sweep direction the AP state (where the resistance is lower) persists over a wider field range (due to exchange bias) compared with the forward field sweep direction. For spin valves with Ho, there is also an asymmetry in $M-H$, although the degree of asymmetry in $R-H$ is somewhat lower than that in $M-H$ for some Ho thickness values.

To quantify the asymmetry, we have estimated from the $M-H$ loops the ratio of the difference between the switching fields of the free and pinned Py layers in the forward and reverse field sweep directions $(\alpha)$ and plotted the dependence of $\alpha$ on $d_{\text {Ho }}$ (red data points) in Fig. 4. We have defined a similar asymmetry parameter $(\beta)$ from the $R-H$ measurements, and the variation of $\beta$ on $d_{\mathrm{Ho}}$ (blue data points) is also plotted in Fig. 4 ( $\beta=1$ means that the peak widths for both field sweep directions are identical). For $d_{\mathrm{Ho}}=2 \mathrm{~nm}$, we do not plot a value of $\beta$ because of a large uncertainty in the width of the AP state in the forward field sweep direction and, therefore, we also do not plot a value of $\alpha$. As $\alpha$ and

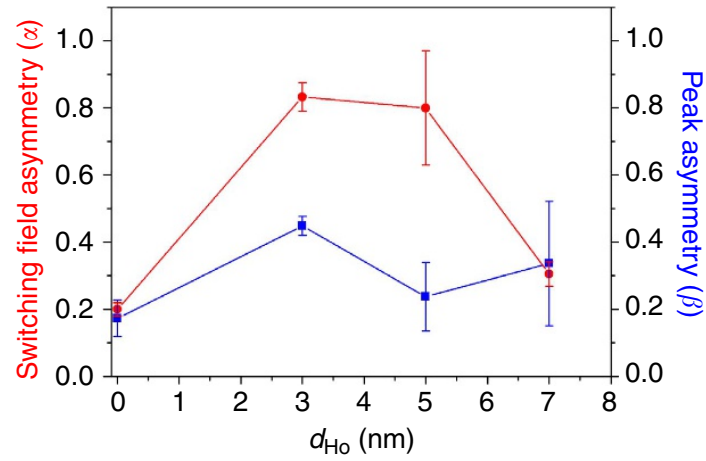

Figure 4 | Asymmetry in peak widths. The switching field asymmetry calculated from the $M-H$ loops $(\alpha)$ and the peak asymmetry from $R-H(\beta)$ are plotted as a function of Ho thickness. The error bars represent 1 s.d. on each side.

$\beta$ are calculated based on magnetic moment and resistance, one of which is volume sensitive and the other a local probe of the magnetization, we do not expect the two values to necessarily match in value.

From Fig. 4 it is clear that the values of $\beta$ remain fairly constant for films with and without Ho, except for the $3 \mathrm{~nm}$ Ho sample. The lower $\beta$-values can be understood as follows: when the Py is in contact with $\mathrm{Ho}$, its switching field range increases; $10 \mathrm{mT}$ for a Py film in contact with a 5-nm-thick layer of Ho compared with $2.5 \mathrm{mT}$ without Ho. In other words, the Py becomes harder to switch and during switching it has an inhomogeneous magnetization distribution over a finite field range. As the superconductivity in the $\mathrm{Nb}$ layer, and thus the resistance of the structure, is very sensitive to the magnetic layer configuration, any inhomogeneity in magnetism will result in a smearing of the resistance peaks and, therefore, to reduced asymmetry. This suggests that the resistance peaks in spin valves with Ho thickness arise from a fundamental modulation of superconductivity in $\mathrm{Nb}$ rather than stray field-induced effects from Py domain walls, as this would result in sharp and symmetric resistance maxima at the switching field of the Py layers. This has been observed in ref. 26 where the $T_{C}$ has a sharp minimum (and therefore, resistance maxima) at the coercive field of Py.

\section{Discussion}

Before considering an explanation for this behaviour involving the generation and diffusion of spin-triplet pairs, we will first discuss possible effects arising from fringing fields ${ }^{26-29}$ and spin imbalance $^{30}$ in $\mathrm{Nb}$, factors that have been advanced as enhancing $T_{\mathrm{C}}$ in the $\mathrm{P}$ state. In the fringing fields scenario, $T_{\mathrm{C}}$ could be suppressed as a consequence of magnetic dipolar coupling between the two $F$ (Py) layers, which introduces flux into the superconductor $(\mathrm{Nb})$. This suppression should be greatest close to the coercive field of the $\mathrm{F}$ layers when the density of the fringing fields from Néel domain walls is maximized ${ }^{28}$. This explanation cannot, however, explain our results, as samples without Ho show a well-defined decrease in $R$ at coercivity and in the AP state, which translates into an enhancement rather than a decrease of $T_{\mathrm{C}}$ (similar results are reported in ref. 9). Even if we assume that exchange coupling with Ho introduces additional domain walls into the Py, given the high saturation field of Ho thin films $(\sim 4 \mathrm{~T})^{19,31}$ the domain configuration would hardly change in the field range investigated and so we would expect to see a monotonic increase in resistance with field rather than a sharp switching as observed in our data. Moreover, the asymmetry in the resistance peaks in $R-H$ for the spin valve without Ho clearly 
shows that $R-H$ reflects the exchange bias observed in the $M-H$ loop, and thus the magnetic state of the spin valve is the underlying factor responsible for the observed resistance peaks.

We now consider the spin-imbalance scenario in $S$ of an $F / S / F$ spin valve ${ }^{30}$, which assumes that spin-polarized quasiparticles flowing in the $\mathrm{F}$ layers scatter into the $\mathrm{S}$. As their transmission probability into the opposite F layer is smaller in the AP state, the spin imbalance induced in the $\mathrm{S}$ is larger than in the $\mathrm{P}$ state. In other words, the spin sensitivity to the relative $\mathrm{F}$ orientation is via quasiparticles. This scenario was originally introduced to explain inverse spin-valve behaviour in $\mathrm{Py} / \mathrm{Nb} / \mathrm{Py}$ structures similar to our own, but more recent work on the same structures ${ }^{26}$ suggest that a fringing field model is actually more probable. In any case, quasiparticle-mediated spin imbalance is necessarily a nonequilibrium effect, and so the effects should be proportional to the voltage drop along the sample. In our experiment we were careful to ensure that the superconducting transition was independent of the applied current and so we can be confident that the observed changes to $T_{\mathrm{C}}$ genuinely reflect the equilibrium properties of the system. Finally, we note that even if spin imbalance occurred, its effect on $T_{\mathrm{C}}$ would be greatest in the spin valves without $\mathrm{Ho}$, as these structures contain fewer quasiparticlescattering interfaces.

This analysis eliminates both fringing field and quasiparticle scattering as a means of explaining the inverse spin-switch behaviour seen in our $\mathrm{Py} / \mathrm{Ho} / \mathrm{Nb} / \mathrm{Ho} / \mathrm{Py}$ devices, and so we consider instead the possibility of spin-one triplet pairs as the transmitters of spin from one $\mathrm{F}$ layer to another.

In the spin valves with Ho, we assume the presence of both singlet pairs and spin-one triplet pairs (which are generated by $\mathrm{Ho}$ and $\mathrm{Ho} / \mathrm{Py}$ interfaces), and so the observed $T_{\mathrm{C}}$ change with magnetic configuration of the spin valves must result from a combination of the standard spin-switch effect and the transmission of spin-one triplet pairs. The standard spin-switch effect, which involves singlet and triplet pairs with zero-spin projection, will always favour a higher $T_{\mathrm{C}}$ in the AP state, as the average exchange field acting on the pairs is lower. This necessarily implies that the increase in $T_{\mathrm{C}}$ observed in the $\mathrm{P}$ state in samples with Ho must be linked to the transmission of spin-one triplet pairs and not to the singlet pairs. Below, we bring forward the most probable explanation of how the triplets control $T_{\mathrm{C}}$.

Consider the cartoon shown in Fig. 5, which illustrates our interpretation. If up-up and down-down triplet pairs were equally involved in proximity coupling with the F layer, then the
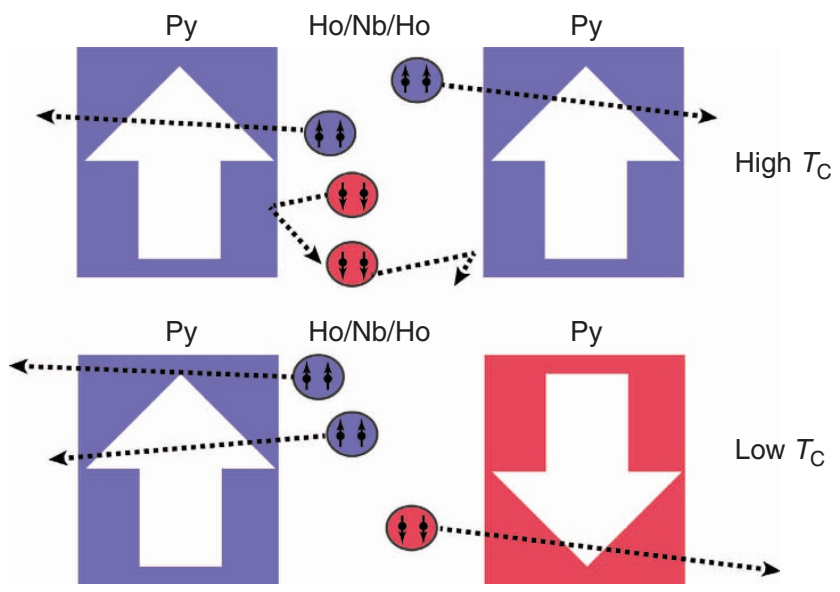

Figure 5 | Illustration of the spin selectivity. A cartoon illustrating the possible behaviour of spin-one triplet pairs in $\mathrm{Py} / \mathrm{Ho} / \mathrm{Nb} / \mathrm{Ho} / \mathrm{Py} / \mathrm{FeMn}$ F-S-F spin valves in the parallel (top) and antiparallel states (bottom).
$T_{\mathrm{C}}$ suppression would be independent of the magnetic orientation of the spin valve. Instead, we assume that at the interface those triplet pairs with spins parallel to the majority spin direction in a Py are more likely to enter it. Spin conservation at the interface therefore implies that opposite spin pairs are more likely to return to the $\mathrm{Nb}$ layer. On the $\mathrm{Nb}$ side, these have a spatial range of at least the singlet coherence length ${ }^{24}$ and so, for the spin valve systems considered here, can interact with the other S-F interface. If this $\mathrm{F}$ layer is $\mathrm{AP}$ to the other $\mathrm{F}$ layer, then the pair has a higher probability of entering it. In other words, the proximity suppression of $T_{\mathrm{C}}$ due to the presence of spin-one triplet pairs, which is governed by the probability of pairs exiting $S$, is enhanced in the AP state in comparison with the P state. This is consistent with our observations. This explanation necessarily requires both the spin-triplet pairs to be able to cross $\mathrm{Nb}$ and that the $\mathrm{F}$ layers are spin selective to the spin of the triplet pairs. In view of theory ${ }^{32,33}$, these assumptions are most likely to be fulfilled in our samples: first, spin selectivity is an intrinsic property of ferromagnets with large spin splitting at the Fermi level ${ }^{34}$ and, second, all triplet components of the condensate induced at the S/F boundary can propagate into a superconductor a distance close to the superconducting coherence length ${ }^{32}$ $\xi_{\mathrm{S}}=\sqrt{\hbar D / k_{\mathrm{B}} T_{\mathrm{C}}}$, which is at best $\sim 30-35 \mathrm{~nm}$ in $\mathrm{Nb}$ where $D$ is the electron diffusivity in $\mathrm{Nb}$ (in our experiment, $D$ is roughly $0.001 \mathrm{~m}^{2} \mathrm{~s}^{-1}$ ). This effect leads to a finite magnetic moment in the superconductor as predicted in refs 32,33 and 35 and observed in ref. 36.

As the magnitude change of MR for the superconducting spin valve without Ho (Fig. 2f) is smaller and of opposite sign to those samples with Ho, we can conclude that the spin selectivity of triplet pairs is dominating the behaviour of $T_{\mathrm{C}}$ over the singlet proximity effect. Exactly how these two opposing behaviours interact is hard to model, as a microscopic theory involving the band structure of the $\mathrm{F}$ layers is required to account for the polarization of the $\mathrm{F}$ layers.

As discussed in the Introduction, spin selectivity is an indispensible requirement for the future development of spin electronics, and so this result represents an important milestone in this field. Furthermore, we believe there is much scope for enhancing the effect of alignment $(\mathrm{P}$ or $\mathrm{AP})$ on $T_{\mathrm{C}}$ by using $\mathrm{F}$ materials with larger spin polarizations. For example, if the $\mathrm{F}$ layers are half-metallic ferromagnets in which case one of the spin channels at the Fermi energy is gapped (meaning 100\% spin polarized), the $T_{\mathrm{C}}$ of an $\mathrm{F}-\mathrm{S}-\mathrm{F}$ spin valve would be greatly suppressed in the AP state if spin-polarized triplet pairs are dominant. Indeed, it is worth noting the most dramatic and reproducible observations of the inverse spin-switch effect have occurred in manganite- $\mathrm{YBa}_{2} \mathrm{Cu}_{3} \mathrm{O}_{7-\mathrm{d}}$-manganite spin valves ${ }^{37,38}$. In both publications, this was assumed to arise from the spinimbalance effect discussed earlier. Although this scenario is more probable in high-temperature superconducting (HTS) systems than in the Nb-based devices discussed here because of the availability of quasiparticle states at all energies (HTS), our results raise the intriguing possibility of the involvement of triplet pairing in this behaviour. There is increasing evidence that spin disorder at manganite-HTS interfaces creates an intrinsic spin-mixer layer ${ }^{39-41}$, and the $100 \%$ spin polarization of the manganites suggests that the dependence of the proximity effect on spin-valve configuration should be significantly higher than in systems involving half-metallic ferromagnets.

\section{Methods}

Film growth. The films were grown by DC magnetron sputter deposition onto unheated oxidized silicon substrates in an ultrahigh vacuum chamber. The chamber was cooled via a liquid nitrogen jacket to achieve a base pressure below $10^{-8} \mathrm{~Pa}$. Substrates rested on a circular table that rotated below stationary 
sputtering targets. All targets were presputtered for at least $15 \mathrm{~min}$ before film growth. The different layers were grown in $1.5 \mathrm{~Pa}$ of $\mathrm{Ar}$ in series by passing the substrates below stationary magnetrons. Growth rates were precalibrated by atomic force microscopy on step edges created by partial lift-off of thin films deposited on patterned substrates. The growth rate of the different layers was controlled by the target power and the speed in which the substrates passed below the stationary targets.

Transport measurements. Resistance measurements of unpatterned samples were performed using a four-point current-bias technique in a pulse-tube cryocooled measurement system with a constant current of $200 \mu \mathrm{A}$. The temperature stability of the system was better than $5 \mathrm{mK}$.

Magnetization measurements. Low-temperature magnetic hysteresis loops of unpatterned films were measured using a vibrating sample magnetometer in which the magnetic field was applied in plane.

\section{References}

1. Buzdin, A. I. Proximity effects in superconductor-ferromagnet heterostructures. Rev. Mod. Phys. 77, 935-976 (2005).

2. Jiang, J. S., Davidović, D., Reich, D. H. \& Chien, C. L. Oscillatory superconducting transition temperature in $\mathrm{Nb} / \mathrm{Gd}$ multilayers. Phys. Rev. Lett. 74, 314-317 (1995).

3. Garifullin, I. A. et al. Re-entrant superconductivity in the superconductor/ ferromagnet V/Fe layered system. Phys. Rev. B 66, 020505(R) (2002).

4. Zdravkov, V. I. et al. Reentrant superconductivity in superconductor/ ferromagnetic-alloy bilayers. Phys. Rev. B 82, 054517 (2010).

5. Tagirov, L. R. Low-field superconducting spin switch based on a superconductor/ferromagnet multilayer. Phys. Rev. Lett. 83, 2058-2061 (1999).

6. Buzdin, A. I., Vedyaev, A. V. \& Ryzhanova, N. N. Spin-orientation-dependent superconductivity in F/S/F structures. Europhys. Lett. 48, 686 (1999).

7. Baladié, I., Buzdin, R. A. N. \& Vedyayev, A. Interplay of superconductivity and magnetism in superconductor/ferromagnet structures. Phys. Rev. B 63, 054518 (2001)

8. Gu, J. Y. et al. Magnetization-orientation dependence of the superconducting transition temperature in the ferromagnet-superconductor-ferromagnet system:CuNi/Nb/CuNi. Phys. Rev. Lett. 89, 267001 (2002).

9. Moraru, I., Pratt, Jr. W. \& Birge, N. O. Observation of standard spin-switch effects in ferromagnet/superconductor/ferromagnet trilayers with a strong ferromagnet. Phys. Rev. B 74, 220507(R) (2006).

10. Bergeret, F. S., Volkov, A. F. \& Efetov, K. B. Long-range proximity effects in superconductor-ferromagnet structures. Phys. Rev. Lett. 86, 4096 (2001).

11. Fominov, Y. V., Golubov, A. \& Kupriyanov, M. Y. Triplet proximity effects in FSF trilayers. JETP Lett. 77, 510 (2003).

12. Fominov, Y. V. et al. Superconducting triplet spin valve. JETP Lett. 91, 308 (2010).

13. Leksin, P. V. et al. Evidence for triplet superconductivity in a superconductorferromagnet spin valve. Phys. Rev. Lett. 109, 057005 (2012).

14. Zdravkov, V. I. et al. Experimental observation of the triplet spin-valve effect in a superconductor-ferromagnet heterostructure. Phys. Rev. B 87, 144507 (2013).

15. Khaire, T. S., Khasawneh, M. A., Pratt, W. P. \& Birge Norman, O. Observation of spin-triplet superconductivity in co-based Josephson junctions. Phys. Rev. Lett. 104, 137002 (2010).

16. Khasawneh, M. A., Khaire, T. S., Klose, C., Pratt, Jr. W. P. \& Birge, N. O. Spintriplet supercurrent in Co-based Josephson junctions. Supercond. Sci. Technol. 24, 024005 (2011)

17. Sprungmann, D., Westerholt, K., Zabel, H., Weides, M. \& Kohlstedt, H. Evidence for triplet superconductivity in Josephson junctions with barriers of the ferromagnetic Heusler alloy $\mathrm{Cu}_{2}$ MnAl. Phys. Rev. B 82, 060505(R) (2010).

18. Robinson, J. W. A., Witt, J. D. S. \& Blamire, M. G. Controlled injection of spintriplet supercurrents into a strong ferromagnet. Science 329, 59-61 (2010).

19. Witt, J. D. S., Robinson, J. W. A. \& Blamire, M. G. Josephson junctions incorporating a conical magnetic holmium interlayer. Phys. Rev. B 85, 184526 (2012).

20. Halász, G. B., Blamire, M. G. \& Robinson, J. W. A. Magnetic-couplingdependent spin-triplet supercurrents in helimagnet/ferromagnet Josephson junctions. Phys. Rev. B 84, 024517 (2011)

21. Usman, I. T. M. et al. Evidence for spin mixing in holmium thin film and crystal samples. Phys. Rev. B 83, 144518 (2011).

22. Anwar, M. S., Veldhorst, M., Brinkman, A. \& Aarts, J. Long range supercurrents in ferromagnetic $\mathrm{CrO}_{2}$ using a multilayer contact structure. Appl. Phys. Lett. 100, 052602 (2012).

23. Klose, C. et al. Optimization of spin-triplet supercurrent in ferromagnetic Josephson junctions. Phys. Rev. Lett. 108, 127002 (2012).

24. Eschrig, M. Spin-polarized supercurrents for spintronics. Phys. Today 64, 4349 (2011).
25. Sosnin, I., Cho, H., Petrashov, V. T. \& Volkov, A. F. Superconducting phase coherent electron transport in proximity conical ferromagnets. Phys. Rev. Lett. 96, 157002 (2006).

26. Steiner, R. \& Ziemann, P. Magnetic switching of the superconducting transition temperature in layered ferromagnetic/superconducting hybrids: spin switch versus stray field effects. Phys. Rev. B 74, 094504 (2006).

27. Stamopoulos, D., Manios, E. \& Pissas, M. Stray-fields-based magnetoresistance mechanism in $\mathrm{Ni}_{80} \mathrm{Fe}_{20}-\mathrm{Nb}-\mathrm{Ni}_{80} \mathrm{Fe}_{20}$ trilayered hybrids. Phys. Rev. B 75, 184504 (2007).

28. Zhu, J., Cheng, X., Boone, C. \& Krivorotov, I. N. Origin of the inverse spin switch effect in superconducting spin valves. Phys. Rev. Lett. 103, 027004 (2009).

29. Flokstra, M., van der Knaap, J. M. \& Aarts, J. Magnetic coupling in superconducting spin valves with strong ferromagnets. Phys. Rev. B 82, 184523 (2010).

30. Rusanov, A. Y., Habraken, S. \& Aarts, J. Inverse spin switch effects in ferromagnet-superconductor-ferromagnet trilayers with strong ferromagnets. Phys. Rev. B 73, 060505(R) (2006).

31. Witt, J. D. S. et al. Strain dependent selection of spin-slip phases in sputter deposited thin-film epitaxial holmium. J. Phys. Condens. Matter 23, 416006 (2011).

32. Bergeret, F. S., Volkov, A. F. \& Efetov, K. B. Induced ferromagnetism due to superconductivity in superconductor-ferromagnet structures. Phys. Rev. B 69, 174504 (2004).

33. Bergeret, F. S., Volkov, A. F. \& Efetov, K. B. Spin screening of magnetic moments in superconductors. Eur. Phys. Lett. 66, 111 (2004).

34. Joachim, S. \& Hans Christoph, S. From Fundamentals to Nanoscale Dynamics. Springer Series in Solid-State Sciences. Vol. 152 (Springer, 2006).

35. Löfwander, T., Champel, T., Durst, J. \& Eschrig, M. Interplay of magnetic and superconducting proximity effects in ferromagnet-superconductor-ferromagnet trilayers. Phys. Rev. Lett. 95, 187003 (2005).

36. Xia, J., Shelukhin, V., Karpovski, M., Kapitulnik, A. \& Palevski, A. Inverse proximity effect in superconductor-ferromagnet bilayer structures. Phys. Rev. Lett. 102, 087004 (2009).

37. Peña, V. et al. Giant magnetoresistance in ferromagnet/superconductor superlattices. Phys. Rev. Lett. 94, 057002 (2005).

38. Pang, B. S. H., Bell, C., Tomov, R. I., Durrell, J. H. \& Blamire, M. G. Pseudo spin-valve behavior in oxide ferromagnet/superconductor/ferromagnet trilayers. Phys. Lett. A 341, 313-319 (2005).

39. Kalcheim, Y., Kirzhner, T., Koren, G. \& Millo, O. Long-range proximity effect in $\mathrm{La}_{2 / 3} \mathrm{Ca}_{1 / 3} \mathrm{MnO}_{3} /(100) \mathrm{YBa}_{2} \mathrm{Cu}_{3} \mathrm{O}_{7-\delta}$ ferromagnet/superconductor bilayers: Evidence for induced triplet superconductivity in the ferromagnet. Phys. Rev. B 83, 064510 (2011).

40. Kalcheim, Y., Millo, O., Egilmez, M., Robinson, J. W. A. \& Blamire, M. G. Evidence for anisotropic triplet superconductor order parameter in halfmetallic ferromagnetic $\mathrm{La}_{0.7} \mathrm{Ca}_{0.3} \mathrm{MnO}_{3}$ proximity coupled to superconducting $\mathrm{Pr}_{1.85} \mathrm{Ce}_{0.15} \mathrm{CuO}_{4}$. Phys. Rev. B 85, 104504 (2012).

41. Visani, C. et al. Equal-spin Andreev reflection and long-range coherent transport in high-temperature superconductor/half-metallic ferromagnet junctions. Nat. Phys. 8, 539-543 (2012)

\section{Acknowledgements}

This work was funded by the Royal Society through a University Research Fellowship ('Superconducting Spintronics') held by J.W.A.R. M.G.B acknowledges funding from the UK EPSRC and the European Commission through an ERC Advanced Investigator Grant ('Superspin'). C.B.S. and R.G.J.S were supported by the Erasmus exchange programme and the Leiden Outbound Grant. The work of F.S.B and A.O. have been supported by the Spanish Ministry of Economy and Competitiveness under Project FIS2011-28851-C02-02. The work of A.O. have also been supported by the CSIC and the European Social Fund under JAE-Predoc programme and the EU-FP 7 MICROKELVIN project (grant number 228464). We are also grateful to Professor Jan Aarts' for scientific input.

\section{Author contributions}

J.W.A.R. had the idea of the experiment and supervised the project. N.B. and C.B.S. prepared the samples and performed the majority of the experiments. R.G.J.S. helped to perform magnetic measurements. A.O. and F.S.B. commented on the paper and performed detailed theoretical calculations. J.W.A.R., N.B. and M.G.B. wrote the paper with help from C.B.S. and F.S.B. All authors read the manuscript.

\section{Additional information}

Competing financial interests: The authors declare no competing financial interests

Reprints and permission information is available online at http://npg.nature.com/ reprintsandpermissions/

How to cite this article: Banerjee, N. et al. Evidence for spin selectivity of triplet pairs in superconducting spin valves. Nat. Commun. 5:3048 doi: 10.1038/ncomms4048 (2014). 